\title{
Optimal design of non-magnetic metamaterial absorbers using visualization method
}

\author{
Xinxin $\mathrm{Xu}^{1}$, Qun Wang ${ }^{1}$, Zhanghong Tang ${ }^{1}$, and Biao Sun ${ }^{2 a)}$ \\ ${ }^{1}$ Electromagnetic Protection and Measurement Institute, College of Material \\ Science and Engineering, Beijing University of Technology, Beijing 100124, China \\ ${ }^{2}$ School of Electrical Engineering and Automation, Tianjin University, \\ Tianjin 300072, China
}

a)sunbiao@tju.edu.cn

\begin{abstract}
This paper presents a design of thin and broadband non-magnetic metamaterial absorbers using circular loop shaped resistive frequency selective surfaces (FSS). A straightforward visual graphic representation analysis which extends the microwave impedance matching theory into wideband metamaterial absorbing structure design is carried out on the key parameters analysis of the absorbers. Absorbers with different layers are analyzed. Numerical computing results are in good agreement with the analyzed ones. The study shows that visual graphic representation analysis could greatly decrease the range of variable. The visualization method is simple and practicable to introduce the working principles of the wideband non-magnetic metamaterial absorbing structure and to derive the best-suited element for wideband absorption.
\end{abstract}

Keywords: metamaterial absorbers, visual graphic, frequency selective surfaces (FSS), wideband

Classification: Electromagnetic theory

\section{References}

[1] L. Lu, S. B. Qu, S. Xia, Z. Xu, H. Ma, J. F. Wang and F. Yu: Acta Phys. Sin. 62 (2013) 013701. DOI:10.7498/aps.62.034206

[2] X. X. Xu, J. J. Jiang, L. Miao, Q. Chen and B. Sun: IEICE Electron. Express 9 (2012) 1408. DOI:10.1587/elex.9.1408

[3] C. Mias and J. H. Yap: IEEE Trans. Antenn. Propag. 55 (2007) 1955. DOI:10.1109/ TAP.2007.900228

[4] J. Mcvay, A. Hoorfar and N. Engheta: Proc. IEEE Int. Symp. on Antennas Propag. (2005) 22.

[5] S. Chakravarty, R. Mittra and N. R. Williams: IEEE Trans. Antenn. Propag. 50 (2002) 284. DOI:10.1109/8.999618

[6] B. A. Munk: Frequency-Selective Surfaces, Theory and Design (Wiley, New York, 2000) 59.

[7] Laird Technologies Company: Products/EMI-Solutions/Specialty-EMI-Solutions/ Microwave-Absorbers (2014) http://www.lairdtech.com. 


\section{Introduction}

In recent years, electromagnetic bandgap materials or metamaterials are suggested to construct ultra-thin absorbers, hybrid radomes, etc [1, 2]. Thin absorbers employing metallic FSS loaded with lumped resistors have been proposed [3]. However, the bandwidths of designed absorbers in these papers are all very narrow [4]. In order to get radar absorbers which present both thin and wideband characteristics, tremendous efforts have been devoted to improving the performance of radar absorbing materials (RAM). Some researchers apply microgenetic algorithm in [5], but maybe too complicated. The designs obtained entirely by numerical optimization needs fast program and computer.

In this paper a novel, thin and wideband absorber is designed from nonmagnetic multilayer structures incorporated with resistive FSS. A novel visual graphic representation analysis of wideband absorbing structure based on smith chart which is convenient for a multianalysis of all the geometric and physical parameter is carried out. Under the guidance of visual graphic representation analysis theory, a broadband absorbing response is obtained through the optimization and calculation. Finally, the numerical method verification of the absorbing structure is presented and compared to the equivalent circuit model predicted ones.

\section{Theory modeling of visual graphic analysis}

\subsection{Equivalent circuit model}

The single layer absorbing panel used in the study consists of one resistive FSS sheet and one grounded spacer [Fig. 1(b)]. The double layer absorbing panel composed of two resistive FSS sheets, one spacer and one grounded spacer [Fig. 1(b)]. FSS is an assembly of identical elements arranged in a two-dimensional infinite array. The elements loaded at their centers have been arranged in a rectangular array with inter-element spacing D. Because a "good" element should be small in terms of wavelength [6], circular loop is chosen as an FSS shape which resonates well before the grating lobes [Fig. 1(a)]. The surface resistance of the resistive FSS was defined as $\mathrm{R}_{\mathrm{S}}$.

Fig. 1(c) shows the equivalent circuit model of the proposed wideband absorber consisting of four sections. Section $\mathrm{A}$ and $\mathrm{C}$ are interconnecting transmission lines. Section B and D consist of resistive FSS. $Y_{g}$ denotes the input admittance of the ground plane. The admittance of the single resistive FSS sheet in air are denoted by $Y_{a 1}$ and $Y_{a 2}$ which can be represented through the reflection coefficient denoted by $\Gamma_{f s s 1}$ and $\Gamma_{f s s 2} . Y_{i n 1}\left(\Gamma_{1}\right), Y_{i n 2}\left(\Gamma_{2}\right)$ and $Y_{i n 3}\left(\Gamma_{3}\right)$ denote the input admittance and reflectivity of the layered absorber structure for normal incidence.

Using this equivalent circuit, the single layer free-space reflectivity of the absorber at normal incidence is given by Eq. (1-4) and the double layer ones is given by Eq. (1-8) where $\mathrm{Z}_{\mathrm{m}}$ and $\varepsilon_{r}$ is the characteristic impedance and the relative permittivity of the ground plane, $\lambda$ is the wavelength of the incidence wave, $Y_{0}$ is the admittance of free-space, $\theta$ is the rotate angle of the transmission line.

$$
Y_{g}=\frac{1}{j Z_{m} \tan \left(2 \pi d \sqrt{\varepsilon_{r}} / \lambda\right)}
$$




$$
\begin{gathered}
Y_{a 1}=-\frac{2 Y_{0} \Gamma_{f s s 1}}{1+\Gamma_{f s s 1}} \\
Y_{a 2}=-\frac{2 Y_{0} \Gamma_{f s s 2}}{1+\Gamma_{f s s 2}} \\
Y_{i n 1}=Y_{g}+Y_{a 1} \\
\Gamma_{1}=20 \log _{10} \frac{Y_{0}-Y_{i n 1}}{Y_{0}+Y_{i n 1}} \\
\Gamma_{2}=\Gamma_{1} e^{-j \theta}, \quad \theta=2 \beta d_{2} \\
Y_{i n 3}=Y_{i n 2}+Y_{a 2} \\
\Gamma_{3}=20 \log _{10} \frac{Y_{0}-Y_{i n 3}}{Y_{0}+Y_{i n 3}}
\end{gathered}
$$

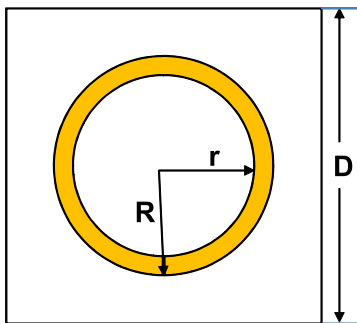

(a)

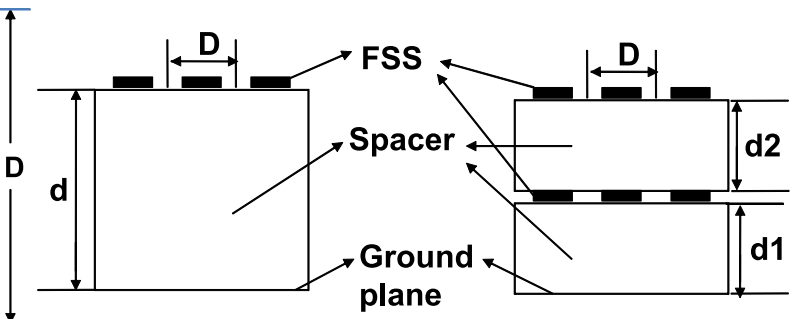

(b)

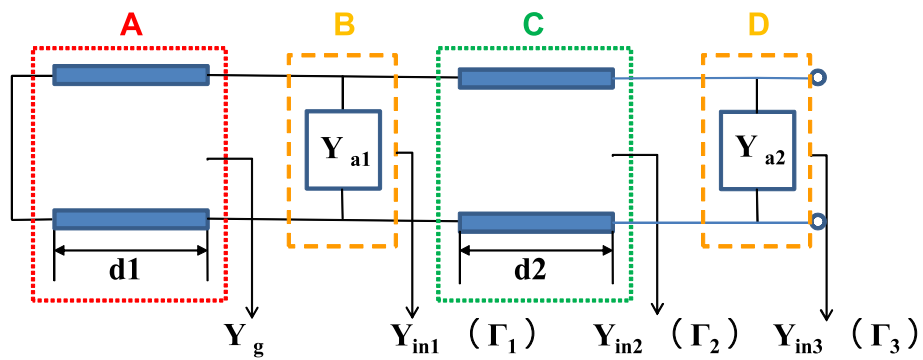

(c)

Fig. 1. (a) Front view of each element in a single resistive FSS sheet. (b) Single and double layer absorbing panel comprising resistive FSS. (c) Equivalent circuit of absorbing panel.

\subsection{Circuit analysis}

The admittance of the equivalent circuit is drawn on the Smith chart [Fig. 2(a)]. It is an ideal wideband impedance matching equivalent circuit model of non-magnetic absorber comprised of a single resistive FSS sheet. The input admittance $Y_{g}$ of the ground plane which can be equal to short transmission line is denoted by curve (1). Similarly, the admittance of the resistive FSS sheet $\mathrm{Y}_{\mathrm{a}}$ is denoted by curve (2). The total admittance of the absorber is simply the sum of $Y_{g}$ and $Y_{a}$ as indicated by curve (3). $Y_{g}$ behaves as an inductor at the lower frequencies in the band of $f_{L}$ to $f_{0}$, and a capacitance at high frequencies in the band of $f_{0}$ to $f_{H}$ as indicated by curve (1) at the rim of the Smith chart. Correspondingly, $Y_{a}$ changes from capacitive to inductive behavior in the band of $\mathrm{f}_{\mathrm{L}}$ to $\mathrm{f}_{\mathrm{H}}$ as indicated by curve (2). At the center frequency $\mathrm{f}_{0}$, the distance between the ground plane and the FSS sheet is approximately $\lambda_{0} / 4$ ( $\lambda_{0}$ is the wavelength against center frequency $\mathrm{f}_{0}$ ) while the 
resistive FSS equivalent circuit resonates. As a result, the resistive FSS sheet is left alone at the center frequency $\mathrm{f}_{0}$. The resistive FSS sheet shows a purely real admittance close to the center of the Smith chart (the best matching point). It is observed that the reactive parts of $Y_{g}$ and $Y_{a}$ to a large degree cancel each other resulting in a location for the band of $\mathrm{f}_{\mathrm{L}}$ to $\mathrm{f}_{\mathrm{H}}$ very close to the center of the Smith chart and leads to a small reflection [Fig. 2(b)].

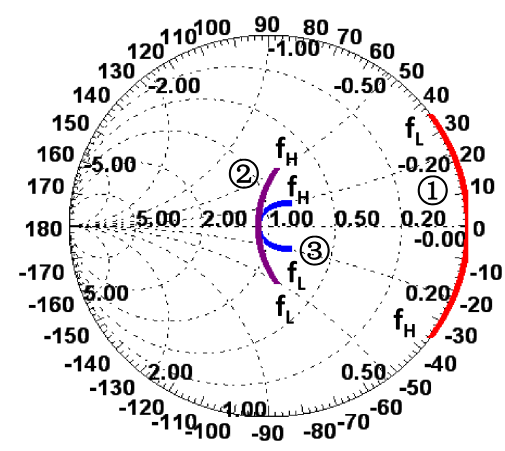

(a)

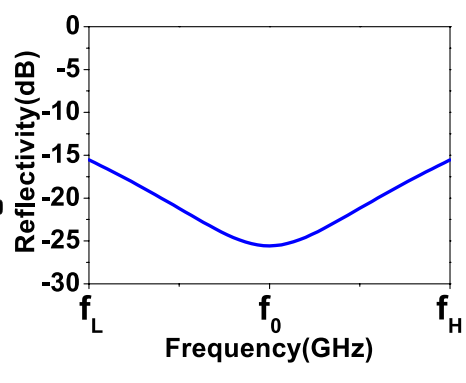

(b)

Fig. 2. (a) Smith chart plots of the ideal impedance matching equivalent circuit. (b) Reflectivity of the ideal single layer absorber model.

\section{Design of non-magnetic absorbers}

\subsection{One layer structure}

With the purpose of obtaining the largest bandwidth design, firstly an attempt is made in the band of $2 \mathrm{GHz}$ to $18 \mathrm{GHz}$. At the center frequency $\mathrm{f}_{0}(10 \mathrm{GHz})$, the electrical thickness is one-fourth wavelength (the distance between the air ground plane and the FSS sheet is $7.5 \mathrm{~mm}$ ). As a result of transmission line law, the electrical thickness will be smaller with higher $\varepsilon_{\mathrm{r}}$ and thereby decrease the thickness of absorbers. However, we also observed some reduction in bandwidth. Thus, the dielectric constant $\varepsilon_{\mathrm{r}}$ should not be increased indiscriminately. Values around 2 to 3 seem to be a good compromise. In order to hold the thickness of absorbers, the optimum dielectric constant is about 2.5 and the electrical thickness is chosen to be $0.25 \lambda_{0}$ (i.e., the mechanical thickness at $10 \mathrm{GHz}$ is equal to $4.75 \mathrm{~mm}$ ). $\mathrm{Y}_{\mathrm{g}}$ is denoted by curve (1) [Fig. 3(a)]. The reactive parts of $Y_{g}$ at $2 \mathrm{GHz}$ and $18 \mathrm{GHz}$ reach to 3 (normalized admittance). The resistive FSS sheet which has an imaginary part of admittance come close to the imaginary parts of $Y_{g}$ and a real part approaches free space admittance $Y_{0}$ should be found to match with $Y_{g}$. At the center frequency $\mathrm{f}_{0}$ $(10 \mathrm{GHz})$, the average radius of the circular loop is chosen to be about $4 \mathrm{~mm}$. In order to match with the endpoints admittance, resistance value is designed as $\mathrm{R}_{\mathrm{S}}=30 \mathrm{ohm} / \mathrm{sq}$. In order to delay the onset of grating lobes, the inter-elemental spacing D should be as small as possible. At the moment a tentative design of $\mathrm{Y}_{\mathrm{a}}$ is obtained by the visual graphic method as shown by curves (2), (3), (4), (5) and (6) which all resonate at $10 \mathrm{GHz}$, it is now perfectly all right to use an optimizer. As a consequence, the absorber can be optimized by micro adjustment of $\mathrm{r}, \mathrm{R}$ and $\mathrm{D}$. Through optimization, when $\mathrm{D}=13 \mathrm{~mm}$, the reactive parts of $Y_{a}$ at $2 \mathrm{GHz}$ and 
$18 \mathrm{GHz}$ reach to 0.54 and -1.14 , the real parts of $\mathrm{Y}_{\mathrm{a}}$ at resonance point is 1.94 . The optimum $Y_{a}$ matched to $Y_{g}$ is denoted by curve (4). By one layer match, the sum of $Y_{a}$ and $Y_{g}$ is shown by curve (3) with the reflectivity shown in Fig. 3(b). The reactive parts of $Y_{g}$ and $Y_{a}$ to a large degree cancel each other resulting in a location from $5 \mathrm{GHz}$ to $15 \mathrm{GHz}$ very close to the center of the Smith chart and leads to a small reflection (blue line in Fig. 3(b)). The reactive parts of $Y_{g}$ in low frequency from $2 \mathrm{GHz}$ to $5 \mathrm{GHz}$ and high frequency from $17 \mathrm{GHz}$ to $18 \mathrm{GHz}$ are high. Therefore, it is difficult to obtain the optimal impedance match between $Y_{a}$ and $Y_{g}$ in the whole band from $2 \mathrm{GHz}$ to $18 \mathrm{GHz}$.

To verify the validity of the equivalent circuit model for non-magnetic absorber, we compare the reflectivity performance of a single layer non-magnetic absorber by the equivalent circuit model against the full-wave finite element method (FEM), as shown in Fig. 3(c). The single layer absorbing structure allow obtaining remarkable performance $(-8 \mathrm{~dB}$ in the band from $4.6 \mathrm{GHz}$ to $16.6 \mathrm{GHz})$ with an overall thickness of $4.75 \mathrm{~mm}$.

It is observed that the results from equivalent circuit model and numerical method are in good agreement. This performance is superior to Salisbury screen, optimized Jaumann screen and the available commercially non-magnetic multilayer structures (see for instance [7]) with the same thickness.

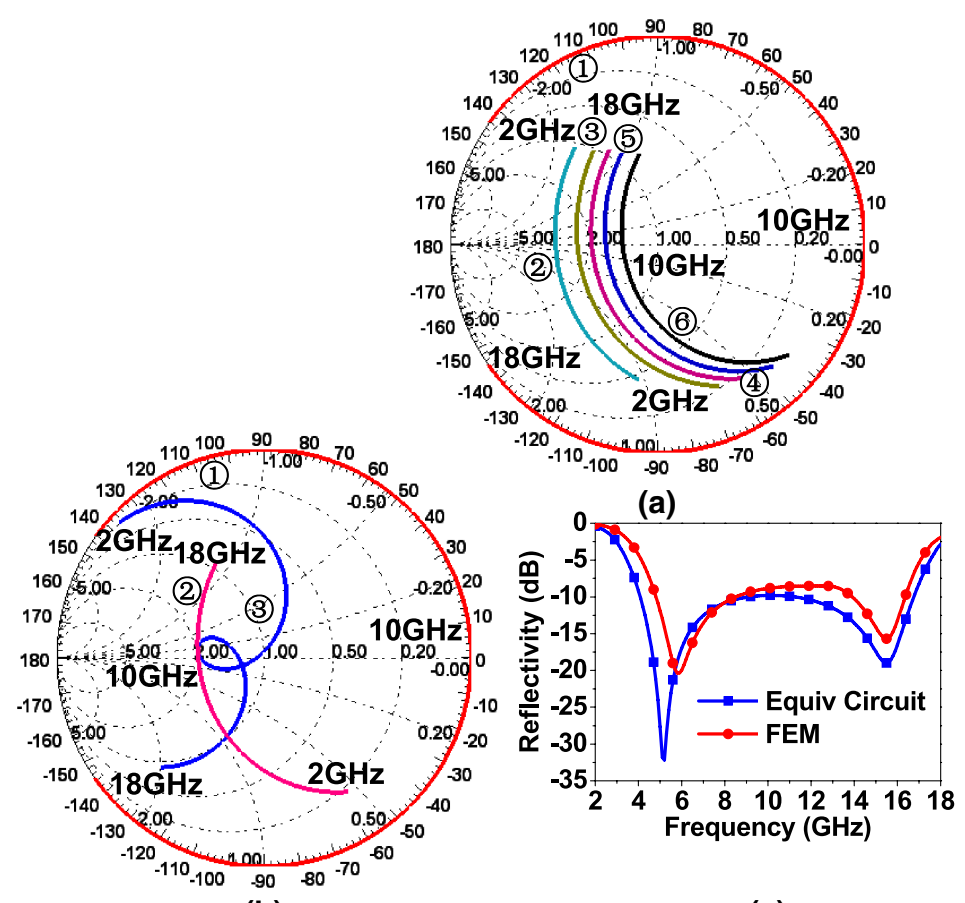

(b)

(c)

Fig. 3. (a) Smith chart plots of the single layer absorber with different D, $r$ and R. (b) Smith chart plots of the single layer absorber. Parameters of the resistive FSS are: Curve (2) Rs $=30 \mathrm{ohm} / \mathrm{sq}$, $\mathrm{D}=13 \mathrm{~mm}$. (c) Reflectivity of the single layer absorber. 
overall thickness to $10 \mathrm{~mm}$. Two different circular loops with the same resonance point are designed as double resistive FSS layer. By the ultra-wideband impedance match design method, $\mathrm{Y}_{\mathrm{a}}$ of the first and second resistive FSS layer are denoted by curves (2) and (5) [Fig. 4(a)]. After matching with the first resistive FSS layer, the sum of curve (1) and curve (2) is shown by curve (3) located on the left of the Smith chart. The low-frequency part of curve (3) is far from the center. Obviously, it is difficult to have ideal result in the low-frequency by curve (3). Traveling through $5 \mathrm{~mm}$ of air layer, the admittance changes to the right of the Smith chart by curve (4). After matching with the second resistive FSS layer by curve (5), curve (6) moves toward the center which has significantly reduced the reflectivity in the low and high frequencies (blue line in Fig. 4(b)).

The reflectivity performance of a double layer non-magnetic absorber was compared by the equivalent circuit model against the full-wave finite element method (FEM), as shown in Fig. 4(b). The double layer absorbing structure allow obtaining remarkable performance $(-8 \mathrm{~dB}$ in the band from $4.1 \mathrm{GHz}$ to $25.5 \mathrm{GHz}$ ) with an overall thickness of $10 \mathrm{~mm}$.

The double layer absorber has greatly enlarged the wave absorption bandwidth and improved the wave absorption performance than single ones.

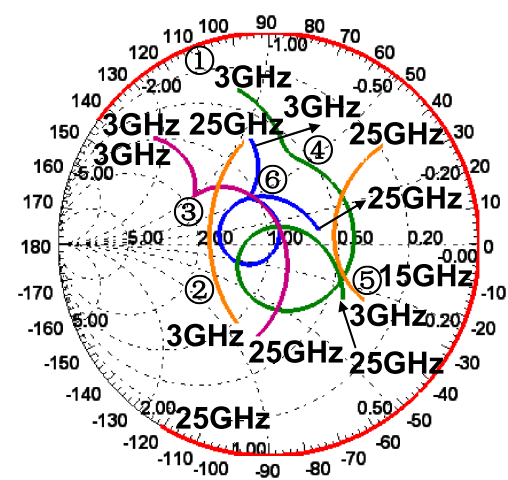

(a)

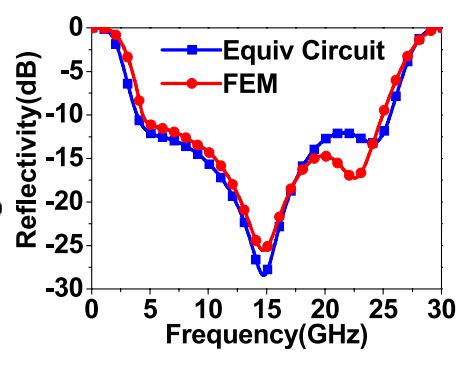

(b)

Fig. 4. (a) Smith chart plots of the double layer absorber. Parameters of the circular loop shaped FSS are: Curve (2) $\mathrm{Rs}=50 \mathrm{ohm} / \mathrm{sq}$, $\mathrm{D}=13 \mathrm{~mm}$; Curve (5) Rs $=30 \mathrm{ohm} / \mathrm{sq}, \mathrm{D}=13 \mathrm{~mm}$. (b) Reflectivity of the double layer absorber.

\section{Conclusions}

In this paper a deep-depth analysis and research are presented on the reflectivity of wideband non-magnetic absorbing structure consists of resistive FSS. The results demonstrate that the use of visual graphic representation analysis method would realize ultra-wideband impedance match in the design of wideband non-magnetic absorbing structure. Compare to the whole process optimized design method, the visual graphic representation analysis method could greatly decrease the range of variable. Once a tentative design is obtained by the visual graphic method, it is perfectly all right to use an optimizer provided that the various parameters are allowed only inside relatively small perturbations. As a result, the visual graphic representation analysis method could greatly improve the efficiency of the design. 
The results also prove that double layer absorber has greatly enlarged the wave absorption bandwidth and improve the wave absorption performance than single ones. The design with a reflectivity less than $8 \mathrm{~dB}$ over a bandwidth exceeding $6: 1$ and thickness less than $0.14 \lambda$ at the lowest frequency is obtained. These research results provide an effective theory instruction for the design of non-magnetic radar absorbing structures.

\section{Acknowledgments}

This work has been supported by the National Natural Science Foundation of China (Grant No. 11104006) and Beijing Postdoctoral Sustentation Fund, China (Grant No. 2014ZZ-31). 